\title{
Decisiones tecnológicas: etiquetado de productos
}

Mónica Anzaldo Montoya*

\author{
Las nanotecnologías plantean una incertidumbre acerca de los beneficios y riesgos \\ para la salud humana y el medio ambiente. Este ensayo tiene el objetivo de reflexionar \\ sobre la importancia de las normas técnicas en las nanotecnologías. El foco de atención \\ se ubica en la reciente publicación de las especificaciones de etiquetado de productos que \\ contienen nanomateriales en el marco de la Organización Internacional de Estándares.
}

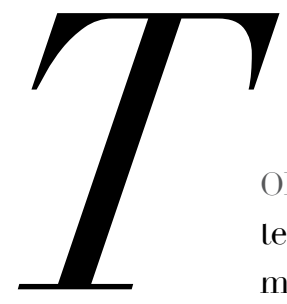

ODOS LOS DÍAS tomamos decisiones tecnológicas. Las decisiones de consumo ahora son decisiones tecnológicas; cuando elegimos un medicamento o una computadora estamos tomando una decisión de carácter tecnológico. Recientemente, ante la necesidad de usar un fotoprotector solar me enfrenté a una serie de disyuntivas para elegir el producto adecuado. En algunos fotoprotectores se informaba sobre los ingredientes, en otros sólo se advertía que no contenían ciertas sustancias.

* El Colegio de San Luis, San Luis Potosí, México. Correo: monicaanzaldo@gmail.com
Por ejemplo, la etiqueta de un fotoprotector decía: "para piel sensible al sol, muy resistente al agua, sin perfume y libre de parabenos". Lo cierto es que las decisiones de consumo se tornan cada día más confusas, difíciles y trascendentales para el entorno socioambiental. Requerimos conjuntar saberes técnicos muy sofisticados para poder identificar si un producto es seguro, si es compatible con otros productos, si es sustentable a lo largo de su ciclo de vida, si es bioacumulable y si está de acuerdo con nuestros valores y estilo de vida (por ejemplo, cuando elegimos alimentos orgánicos). El establecimiento de normas o estándares nacionales e internacionales se ha vuelto un instrumento muy importante para la introducción y la estabilización de las tecnologías en la sociedad. Se considera que una 
tecnología o artefacto se ha estabilizado cuando los grupos que intervienen en el diseño y construcción han disminuido las interpretaciones o controversias sobre el funcionamiento. El término estabilización fue propuesto por Wiebe Bijker.'

De acuerdo con la Organización Internacional de Estándares (ISO, por sus siglas en inglés), una norma es un documento establecido por consenso y aprobación de un organismo reconocido que proporciona los requisitos, especificaciones, lineamientos y características que podrán ser utilizados para asegurar que los materiales, productos, procesos y servicios sean adecuados para su propósito. Bajo esta definición, los estándares técnicos son la materialización de acuerdos entre diferentes expertos para que funcione la tecnología. Estos acuerdos ejercen una influencia en el comercio mundial de mercancías y, como se acaba de señalar, tienen efectos trascendentes en nuestras vidas y en el medio ambiente.

Las nanotecnologías aún son tecnologías en proceso de estabilización. Sus aplicaciones han generado acalorados debates científicos, pues hay quienes advierten sobre la incertidumbre de los efectos de los nanomateriales en la salud y el medio ambiente, en tanto otros señalan que estas tecnologías no son más peligrosas que las ya conocidas. En este contexto, el establecimiento de estándares se ha vuelto un asunto crítico para que estas tecnologías se desplieguen a un mayor número de sectores. Este ensayo tiene el objetivo de presentar brevemente la importancia de la normalización internacional de las nanotecnologías con énfasis en la especificación del etiquetado. El etiquetado de productos derivados de tecnologías emergentes se caracteriza por ser un asunto altamente debatido. Ninguna empresa quiere generar percepciones negativas sobre los productos en los que ha invertido millones de dólares. Si traemos a cuenta lo que sucedió con los organismos genéticamente modificados en países como Francia o México, una percepción orientada hacia los riesgos puede crear una importante barrera para el mercado y enturbiar planes de inversión.

\section{Situación de la normalización}

DE LAS NANOTECNOLOGÍAS

EN EL MARCO DE LA ISO

En 2005, la ISo conformó el comité técnico número 229 para la normalización de las nanotecnologías (en adelante ISO/TC 229), el cual está conformado por los insti- tutos de metrología de los países miembros de esa organización. Hasta el momento, 48 países integran ese comité, entre los que se incluye México. El ISO/TC 229 se creó para que quienes participan ahí acuerden el contenido de normas o estándares internacionales que ayuden a potenciar el uso de las herramientas de las nanotecnologías, superando las limitantes técnicas que obstaculicen su mercado, como la falta de un lenguaje común que facilite el intercambio de nanomateriales, la falta de métodos de medición y caracterización y la presión por atender el controvertido tema de la toxicidad de los nanomateriales. En términos macrosociales, el ISO/TC 229 fue creado para poner en marcha los acuerdos político-económicos que aceleren el desarrollo de esta tecnología.

Para poner en marcha los acuerdos, el ISO/TC 229 sirve como lugar de encuentro entre actores gubernamentales y empresariales, pues cada país miembro conforma en su territorio un comité espejo que sirve de puente para que los actores públicos y privados inserten sus intereses en la redacción de las normas. En los proyectos del comité también participan varios organismos internacionales como la Organización para la Cooperación y el Desarrollo Económicos (OCDE), la Asociación de Industrias de Nanotecnología (NIA, por sus siglas en inglés) de Europa y organizaciones no gubernamentales (ONG) interesadas en los derechos del consumidor, como la European Association for the Coordination of Consumer Representation in Standardization (ANEC) y la European Environmental Citizens Organization for Standarization (ECOS).

La velocidad en la producción de normas no es al parecer la más deseada. Tres años después de haberse creado el ISO/TC 229 se publicó el primer documento normativo para las nanotecnologías. No se trató de una norma como tal, sino de una Especificación Técnica, la número 27687 , Nanotechnologies, terminology and definitions for nano-objects. Nanoparticle, nanofibre and nanoplate, en la cual se definen i2 términos de las nanotecnologías. El listado de documentos publicados por el ISO/TC 229 está disponible en: http://www.iso.org/iso/ home/store/catalogue_tc/catalogue_tc_browse.htm? commid=38ז983\&includesc $=$ true $\&$ published $=$ on.

Hasta enero de 20I4, el ISO/TC 229 había publicado 40 documentos: tres normas internacionales, 27 especificaciones técnicas y Io reportes técnicos. La escasa producción de normas refleja que los temas tratados son controvertidos, en algunos casos requieren de mayor investigación científica. Decimos esto porque en el lenguaje de los comités de la Iso, la norma es un documento 


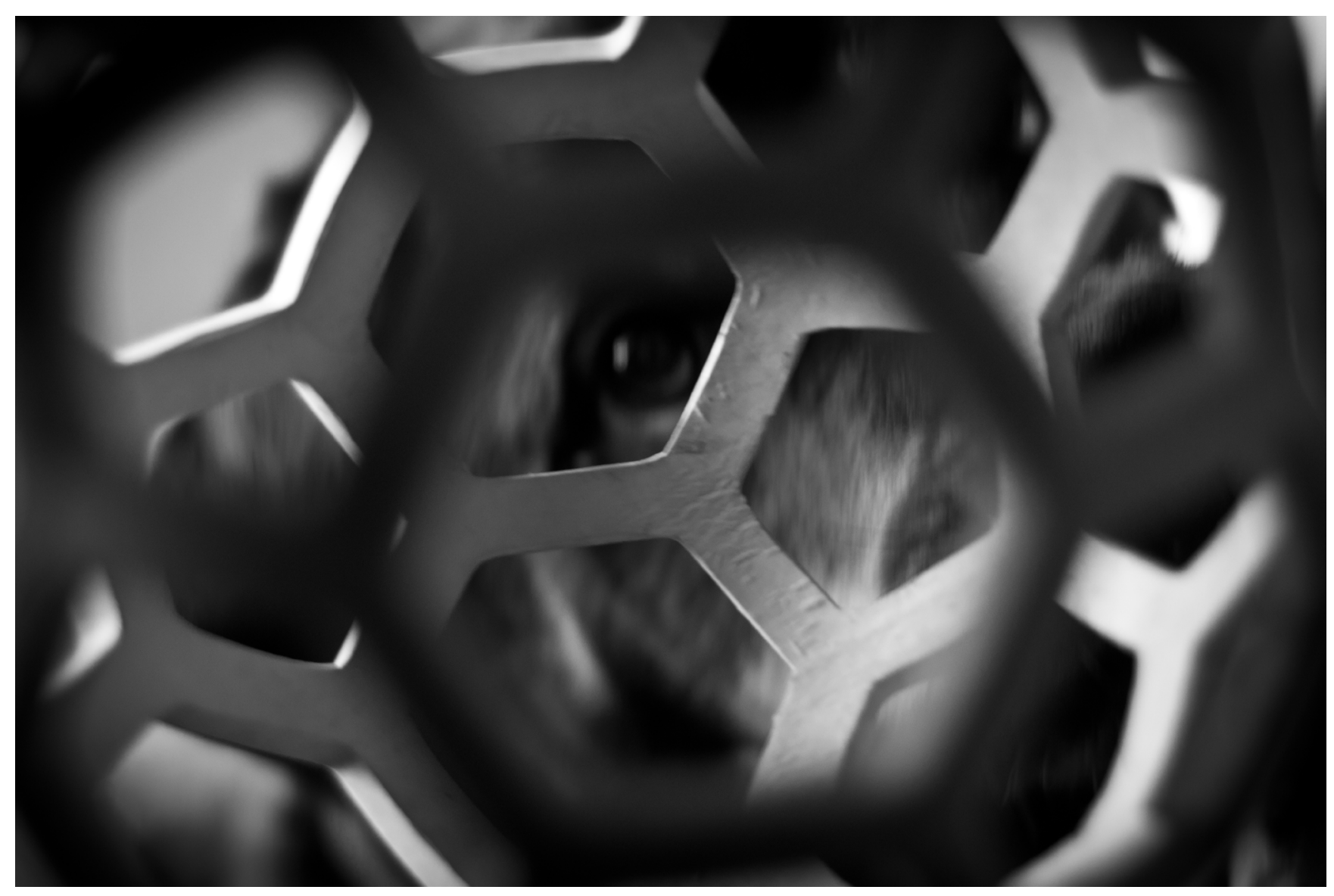

cuyo contenido logró mayor consenso y es más estable en el tiempo que una especificación o reporte técnico.

\section{LA INCÓMODA DEMANDA DEL ETIQUETADO}

En noviembre de 20I3, el ISO/TC 229 publicó la Especificación técnica de etiquetado. El etiquetado es una demanda muy importante para los consumidores informados de sus derechos. Es un tema muy controvertido en la regulación de las nuevas tecnologías porque involucra un mensaje directo a los ciudadanos sobre los beneficios y riesgos de la misma. Visto de otra manera, la etiqueta es un mensaje que llega al grupo de interés más numeroso y relevante para la percepción de cualquier tecnología.

La publicación de la especificación del etiquetado en el ámbito de la ISO no estuvo exenta de escollos, lo cual se refleja en que el documento tardó tres años en aprobarse y que su alcance fue renegociado. Una primera versión del documento circuló en 2010 con el título de Guidance on the labelling of manufactured nanoobjects and products containing manufactured nano-objects, mientras que la versión publicada en 2013 se titula Guidance on the voluntary labelling of consumer products containing manufactured nano-objects. Una presentación del Centre for Bioethics and Emerging Technologies compara varias iniciativas sobre el etiquetado de productos con nanomateriales. ${ }^{2}$ Tal como se observa, la guía de etiquetado vigente enfatiza el carácter voluntario de la especificación y se refiere únicamente al etiquetado de productos de consumo final que contienen nano-objetos, es decir, aquellos destinados al uso personal y no al de productos que son objeto de comercio entre proveedores y fabricantes.

En el ámbito de controversia e incertidumbre sobre los efectos de los nanomateriales en la salud y el medio ambiente, el etiquetado es un instrumento que informa del riesgo y permite a los consumidores ejercer su derecho a elegir de forma informada. En ese sentido, la aprobación de la especificación técnica del etiquetado representa un paso adelante en la difusión de las nanotecnologías en la sociedad; es un instrumento efectivo si la información es precisa, en este caso, si la etiqueta indica el tipo de sustancia que contiene el producto; no basta con que se diga que contiene nanomateriales, sino que se señale el tipo de nanomaterial, la cantidad y los potenciales riesgos. Quienes se oponen al etiquetado de productos que contienen nanomateriales argumentan que el etiquetado sólo alarmará al público y lo confundirá. 
El etiquetado de productos, no obstante, no sustituye otras medidas que las empresas y gobiernos deben tomar para advertir al público sobre los usos de los productos. Por ejemplo, la elaboración de hojas de seguridad, el cumplimiento de registro ante la autoridad, la evaluación de riesgos antes de la comercialización de un producto, etcétera. Las normas de etiquetado generadas por la ISO tienen sus limitantes, porque las características de las etiquetas deben ser aplicables para todos los países miembros, y ese acuerdo resultadifícil de alcanzar.

Desde una perspectiva crítica, el etiquetado de los productos no responde completamente a las necesidades de seguridad de la sociedad moderna, sobre todo con la complejidad de las nuevas tecnologías. Con el sencillo ejemplo dado al inicio de este ensayo se hace evidente que la mera información no es suficiente, pues se traslada al consumidor la responsabilidad de utilizar un producto cuya etiqueta contiene información limitada y difícil de entender. Esta situación se explica bien bajo lo que el sociólogo Ulrich Beck denomina "irresponsabilidad organizada". ${ }^{3}$ Este autor señala que cuando la responsabilidad es colectivizada se diluye y entonces resulta que nadie es responsable y no hay compensación por el daño. Esta reflexión es pertinente con respecto a la cuestión del etiquetado, si traemos a cuenta el componente de incertidumbre sobre los riesgos de los nanomateriales.

\section{Reflexiones finales}

México participa activamente en la redacción de normas internacionales para las nanotecnologías, pues es miembro con derecho a voto en el ISO/TC 229. No obstante, existe una enorme brecha en el número de documentos adoptados. Recientemente se publicó en el Diario Oficial de la Federación la vigencia de cinco normas mexicanas para las nanotecnologías: 1) caracterización de muestras de nanotubos de carbono de pared múltiple (NTCPM);2) caracterización de nanotubos de carbono de una pared (NTCIP) mediante espectroscopía de fotoluminiscencia en el infrarrojo cercano (EFL-IRC); 3) terminología y definiciones para nano-objetos -Nanopartícula, nanofibra y nanoplaca; 4) vocabulario Parte ı: Conceptos básicos; 5) vocabulario -Parte 3: Nano-objetos de carbono. ${ }^{4}$

Entre dichas normas no se encuentra la especificación para el etiquetado ni la serie de documentos que guían la protección a la salud de los trabajadores (el documento se titula Occupational risk management applied to engineered nanomaterials. Part 1:Principles and approaches). Con lo dicho, no se pretende señalar que México tendría que limitarse a adoptar la regulación voluntaria internacional para las nanotecnologías; a nivel nacional el Estado mexicano tendría que estar formulando estrategias de evaluación y seguimiento para los productos de las nanotecnologías.

La reflexión final que queremos hacer es sobre la situación de atraso en que se encuentra la participación del público en la toma de decisiones tecnológicas, incluyendo la participación en la redacción de normas, los derechos del consumidor y del ciudadano en el campo de las nanotecnologías, un tema casi esotérico. Mientras escribimos estas líneas sigue creciendo el mercado de productos con nanomateriales sin darnos el derecho a decidir sobre lo que comemos, usamos o vertemos en el agua.

\section{- notas $\cdot-$}

'Wiebe Bijker (2005), “¿Cómo y por qué es importante la tecnología?", Redes, II, 2, pp. 19-53.

${ }^{2}$ http://www.bioethics.ac.uk/cmsfiles/files/resources/ PPointPDFs/G-Hunt-PP-slides-28-o2-II.pdf.
${ }^{3}$ Ulrich Beck (1998), La sociedad del riesgo. Hacia una nueva modernidad, traducción de Jorge Navarro, Daniel Jiménez y María Rosa Borrás, Barcelona, Paidós Básica.

${ }^{4}$ Disponibles en: http://www.economia-nmx.gob.mx/normasmx/consulta.nmx. 